\title{
Evaluation of $(1+\alpha)$ Fractional-Order Approximated Butterworth High-Pass and Band-Pass Filter Transfer Functions
}

\author{
David Kubanek $^{1}$, Todd Freeborn ${ }^{2}$, Jaroslav Koton ${ }^{1}$, Norbert Herencsar ${ }^{1}$ \\ ${ }^{I}$ Department of Telecommunications, Brno University of Technology, \\ Technicka 12, 61600 Brno, Czech Republic \\ ${ }^{2}$ Department of Electrical and Computer Engineering, University of Alabama, \\ PO Box 870286, Tuscaloosa, USA \\ kubanek@feec.vutbr.cz
}

\begin{abstract}
This paper summarizes multiple cases of highpass (HP) and band-pass (BP) analogue filter transfer functions with fractional order $1<(1+\alpha)<2$. All HP and BP transfer functions are evaluated against the magnitude characteristics of ideal Butterworth responses when coefficients previously determined to approximate fractional-order (FO) Butterworth low-pass (LP) transfer functions are utilized. Comparisons of the simulated FO HP and BP responses against the ideal Butterworth responses are presented, with a least squares error analysis applied to determine the transfer functions that best approximate the Butterworth response for both HP and BP FO filters.
\end{abstract}

Index Terms-Active filters; Butterworth approximation; Fractional calculus; Fractional-order filters; Transfer functions.

\section{INTRODUCTION}

The process of applying an electronic filter to remove unwanted frequency characteristics from an input signal is one of the most often used operations in signal processing. Commonly, these filter circuits are integer order (i.e. $1^{\text {st }}, 2^{\text {nd }}$, $3^{\text {rd }}$ ), but recently filter circuits with a fractional-order (FO) (i.e. non-integer orders, 1.1, 2.7, 6.4, etc.) have been introduced [1]-[10]. These FO filters import concepts from fractional calculus and allow for the development of FO systems that offer better performance and flexibility compared to their integer-order counterparts [11], [12]. The order of the filter $(n)$ defines the slope of the magnitude characteristics in the stop-band of a designed filter. For example, in the case of low-pass (LP) and high-pass (HP) filters the stop-band slopes are $-20 n \mathrm{~dB} / \mathrm{dec}$ and $+20 n \mathrm{~dB} / \mathrm{dec}$, respectively. When extending filters to $\mathrm{FO}$ with orders that are positive real numbers, the stop-band magnitude characteristics become $-20(n+\alpha) \mathrm{dB} / \mathrm{dec}$ and $+20(n+\alpha) \mathrm{dB} / \mathrm{dec}$ for the LP and HP responses,

Manuscript received 6 October, 2017; accepted 28 February, 2018.

This article is based upon work from COST Action CA15225, a network supported by COST (European Cooperation in Science and Technology). Research described in this paper was financed by the National Sustainability Program under grant LO1401 and by the Czech Science Foundation under grant no. 16-06175S. For the research, infrastructure of the SIX Center was used. respectively, where $n$ is the integer order and $0<\alpha<1$ is the fractional component. This increases the range of attenuation values in the filter magnitude response that are realizable using the FO filters over their integer-order counterparts.

A FO LP filter with the order $1<(1+\alpha)<2$ can be designed using the transfer functions given by [4]:

$$
\begin{gathered}
H_{1+\alpha}^{\mathrm{LP}-\mathrm{A}}(s)=\frac{k_{\mathrm{A} 1}}{s^{1+\alpha}+s^{\alpha} k_{\mathrm{A} 2}+k_{\mathrm{A} 3}} \\
H_{1+\alpha}^{\mathrm{LP}-\mathrm{B}}(s)=\frac{k_{\mathrm{B} 1}}{s^{1+\alpha}+s k_{\mathrm{B} 2}+k_{\mathrm{B} 3}},
\end{gathered}
$$

where $s$ is the complex variable and the coefficients $k_{\mathrm{A} 1}, k_{\mathrm{A} 2}$, $k_{\mathrm{A} 3}, k_{\mathrm{B} 1}, k_{\mathrm{B} 2}, k_{\mathrm{B} 3}$ are determined based on the desired shape of the frequency characteristics (cut-off frequency, passband gain, pass-band ripple, stop-band slope, etc.). The transfer functions (1) and (2) can be implemented by traditional second-order filter topologies where the standard capacitor is replaced by FO capacitor with impedance $Z=$ $1 /\left(s^{\alpha} C\right)$, where $C$ is a pseudo capacitance with its unit $\mathrm{F} \cdot \sec ^{\alpha-1}[4]$.

To design a FO LP response with a maximally flat passband, i.e. a Butterworth-like response, the coefficients for (1) and (2) were previously determined through application of an optimization routine to approximate the Butterworth pass-band [4]. This method applied a nonlinear least squares optimization routine to find the coefficients of the functions (1) and (2) that best approximated the first-order LP Butterworth function with pole frequency $1 \mathrm{rad} / \mathrm{s}$ in the passband from $\omega=0.01 \mathrm{rad} / \mathrm{s}$ to $1 \mathrm{rad} / \mathrm{s}$. The interpolated equations that describe the coefficients $k$ as functions of $\alpha$, as presented in [4], are repeated below:

$$
\begin{gathered}
k_{\mathrm{A} 1}=1, \\
k_{\mathrm{A} 2}=1.008 \alpha^{2}+0.2867 \alpha+0.2366, \\
k_{\mathrm{A} 3}=0.2171 \alpha+0.7914 \\
k_{\mathrm{B} 1}=1, \\
k_{\mathrm{B} 2}=-0.4838 \alpha^{2}+2.023 \alpha+0.0104,
\end{gathered}
$$




$$
k_{\mathrm{B} 3}=-0.0992 \alpha^{2}+0.0989 \alpha+1.004 .
$$

It should be noted that the coefficients for LP FO transfer functions have also been determined using similar methods to approximate Chebyshev [5], inverse-Chebyshev [6], and arbitrary quality factor [7] magnitude responses.

The aim of this paper is to present both FO HP and bandpass (BP) filter transfer functions and analyse their magnitude responses using various coefficients in these functions. This topic has not yet received much attention, with many FO filter designs for $\mathrm{HP}$ and $\mathrm{BP}$ transfer functions using the same coefficients as for the LP function; under the presumption that the HP or BP transfer function will have the properties corresponding to the original LP (e.g. maximally flat magnitude response, the same characteristic frequency, pass-band gain, etc.) [8], [10]. This needs to be investigated in detail to understand if this assumption is valid and provides the motivation and novelty of this work.

This paper is organized as follows: Section II details the theoretical description of obtaining various forms of FO HP transfer functions. Section III numerically evaluates the accuracy of these HP transfer functions compared to the first-order Butterworth HP function. With Section IV presenting several forms of FO BP transfer functions that are numerically evaluated in Section V. Finally, Section VI concludes the paper suggesting the extended possibilities of FO transfer function design using numerical optimization methods.

\section{FRACTIONAL-ORDER HP TRANSFER FUNCTIONS}

The following cases describe three forms for HP transfer functions that can be obtained through transformation of the FO LP functions (1) and (2).

\section{A. Case HP1}

The first case of HP transfer functions is obtained by multiplying (1) and (2) by $s^{1+\alpha}$, which yields:

$$
\begin{gathered}
H_{1+\alpha}^{\mathrm{HP} 1-\mathrm{A}}(s)=\frac{s^{1+\alpha} k_{\mathrm{A} 1}}{s^{1+\alpha}+s^{\alpha} k_{\mathrm{A} 2}+k_{\mathrm{A} 3}}, \\
H_{1+\alpha}^{\mathrm{HP} 1-\mathrm{B}}(s)=\frac{s^{1+\alpha} k_{\mathrm{B} 1}}{s^{1+\alpha}+s k_{\mathrm{B} 2}+k_{\mathrm{B} 3}} .
\end{gathered}
$$

The HP transfer function given by (9) was previously used in [8] and is usually available when another input or output is selected in a LP filter implemented by a multi-loop feedback structure. Let us consider the transfer function coefficients given by (3)-(8), i.e. the Butterworth-like approximation of the original LP function. Due to the unity values of the coefficients $k_{\mathrm{A} 1}$ and $k_{\mathrm{B} 1}$ the high-frequency pass-band gain of (9) and (10) is unity, i.e. $0 \mathrm{~dB}$. On the contrary, the LP functions (1) and (2) provide pass-band gains of $1 / k_{\mathrm{A} 3}$ and $1 / k_{\mathrm{B} 3}$ respectively, which is not unity for most values of $\alpha$. In these cases the LP and the transformed HP functions are not symmetrical. Thus, this transformation is not the optimal LP to HP transformation for the FO transfer functions of the form (1) and (2) that maintains the lowest deviation from the first-order Butterworth function. The numerical analyses in the Section III will quantify these differences and their errors.

\section{B. Case HP2}

Similar to the HP1 case, another method to obtain a HP transfer function from (1) and (2) multiplies them by $s^{1+\alpha}$ and (assuming $k_{\mathrm{A} 1}=1$ and $k_{\mathrm{B} 1}=1$ ) applies an additional gain correction $1 / k_{\mathrm{A} 3}$ and $1 / k_{\mathrm{B} 3}$, respectively:

$$
\begin{gathered}
H_{1+\alpha}^{\mathrm{HP} 2-\mathrm{A}}(s)=\frac{s^{1+\alpha} / k_{\mathrm{A} 3}}{s^{1+\alpha}+s^{\alpha} k_{\mathrm{A} 2}+k_{\mathrm{A} 3}}, \\
H_{1+\alpha}^{\mathrm{HP} 2-\mathrm{B}}(s)=\frac{s^{1+\alpha} / k_{\mathrm{B} 3}}{s^{1+\alpha}+s k_{\mathrm{B} 2}+k_{\mathrm{B} 3}} .
\end{gathered}
$$

The magnitude properties of (11) and (12) are also evaluated in Section III.

\section{Case HP3}

The third HP transfer function considered in this work applies the traditional substitution of the complex variable $s$ by $1 / s$. Applying this transformation to (1) and (2) yields the following transfer functions:

$$
\begin{aligned}
& H_{1+\alpha}^{\mathrm{HP} 3-\mathrm{A}}(s)=\frac{s^{1+\alpha} k_{\mathrm{A} 1}}{s^{1+\alpha} k_{\mathrm{A} 3}+s k_{\mathrm{A} 2}+1}, \\
& H_{1+\alpha}^{\mathrm{HP} 3-\mathrm{B}}(s)=\frac{s^{1+\alpha} k_{\mathrm{B} 1}}{s^{1+\alpha} k_{\mathrm{B} 3}+s^{\alpha} k_{\mathrm{B} 2}+1} .
\end{aligned}
$$

The pass-band gains of (13) and (14) are $1 / k_{\mathrm{A} 3}$ and $1 / k_{\mathrm{B} 3}$ respectively, when considering the unity values of coefficients $k_{\mathrm{A} 1}$ and $k_{\mathrm{B} 1}$, see (3) and (6). The denominator coefficients of (13) and (14) are interchanged compared to (1) and (2) with an additional change of the exponent of $s$ in the middle terms of the denominators. Now, while this transformation does provide symmetrical HP and LP magnitude characteristics with respect to the frequency at $1 \mathrm{rad} / \mathrm{s}$, the differences in the denominator coefficients prevent the simultaneous realization of LP and HP filters with a single topology without adjusting the circuit elements and their values.

\section{EVALUATION OF THE HP TRANSFER FUNCTIONS}

To evaluate each of the HP transfer functions presented in Section II, their magnitude frequency characteristics compared to the magnitude of first-order Butterworth HP transfer function $s /(s+1)$ are given in Fig. 1 and Fig. 2. Figure 1 depicts the characteristics of the functions (9), (11), and (13) with $\alpha=0.5$ and the coefficients determined using (3)-(5), with Fig. 2 presenting (10), (12), and (14) again with $\alpha=0.5$ and the coefficients according to (6)-(8). For comparison, the first-order Butterworth HP transfer functions are also shown in both figures as a dashed line.

It is apparent from Fig. 1 and Fig. 2 that each of the FO HP filters do not realize the same magnitude characteristics. As the coefficients (3)-(8) for approximating the first-order LP transfer function in the range 0.01 to $1 \mathrm{rad} / \mathrm{s}$ were used, it 
is fair to evaluate the deviation of the FO HP magnitudes from the first-order one between the reciprocal frequencies 1 to $100 \mathrm{rad} / \mathrm{s}$.

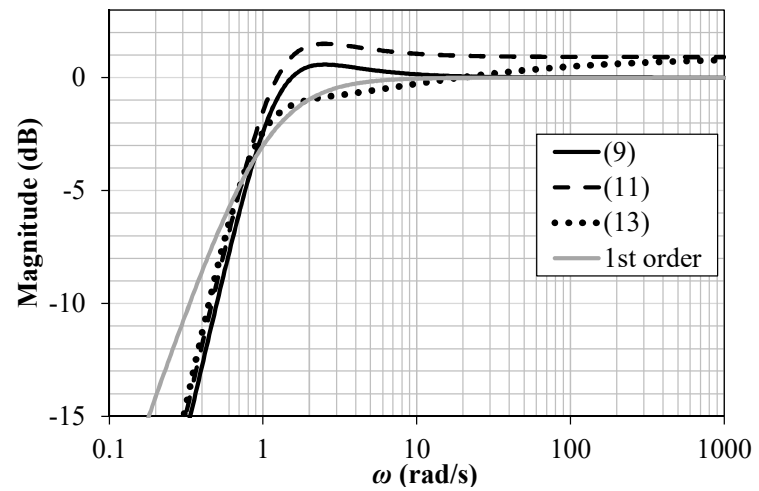

Fig. 1. Magnitude characteristics of (9), (11), (13) with $\alpha=0.5$ compared to the first-order Butterworth HP response.

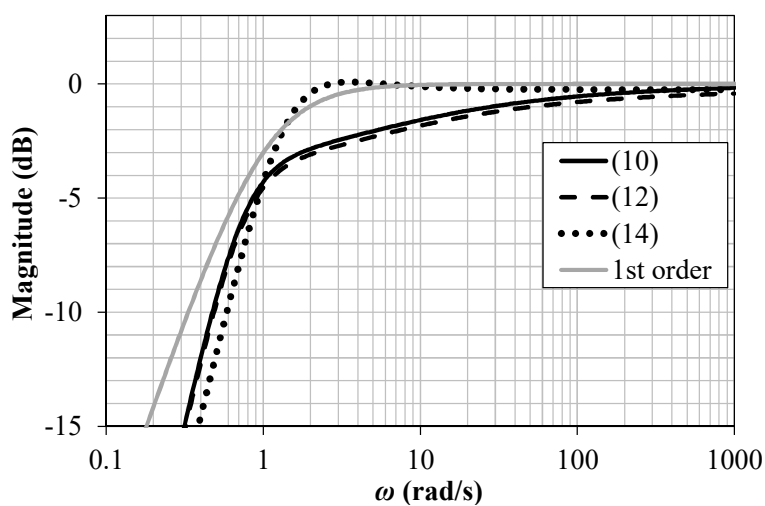

Fig. 2. Magnitude characteristics of (10), (12), (14) with $\alpha=0.5$ compared to the first-order Butterworth HP response.

In Fig. 1 the solid curve presenting (9) has a larger gain than the ideal Butterworth response in the whole frequency range of interest and exhibits peaking above the cut-off frequency. This is also true for the dashed trace which presents (11) and has an even greater gain deviation from the ideal Butterworth response than (9). On the other hand, the dotted curve representing (13) exhibits both positive and negative errors in the range $1 \mathrm{rad} / \mathrm{s}$ to $100 \mathrm{rad} / \mathrm{s} \mathrm{compared} \mathrm{to}$ the Butterworth response, but is still the most accurate of the three cases. Thus, the transformation $s$ to $1 / s$ used in the HP3 case is the best choice for transforming FO LP to HP in this specific frequency band when the LP coefficients are used for $\alpha=0.5$. Note that when considering even higher frequencies the transfer function (9) has the lowest error as its magnitude tends to $0 \mathrm{~dB}$.

Observing the responses in Fig. 2, again the dotted trace representing (14) from the HP3 case provides the best approximation, with the responses given by (10) and (12) showing significant deviation from the Butterworth response; with significantly lower gain in the band from $1 \mathrm{rad} / \mathrm{s}$ to $100 \mathrm{rad} / \mathrm{s}$.

To be able to evaluate the accuracy of the HP transfer functions for any $\alpha$ from the range between zero and one, the least square errors (LSEs) between magnitudes of these functions and the first-order Butterworth one were computed as shown in the following Fig. 3 and Fig. 4. These LSEs were computed using

$$
\mathrm{LSE}=\sum_{i=1}^{n}\left[\left|H_{1+\alpha}\left(\omega_{i}\right)\right|-\left|H_{\mathrm{int}}\left(\omega_{i}\right)\right|\right]^{2},
$$

where $\left|H_{1+\alpha}\left(\omega_{i}\right)\right|$ is the FO magnitude at frequency $\omega_{i}$, $\left|H_{\text {int }}\left(\omega_{i}\right)\right|$ is the first-order HP Butterworth magnitude at frequency $\omega_{i}$, and $n$ is the total number of compared frequency points. In this case, $n=100$ and the datapoints were selected from the range $1 \mathrm{rad} / \mathrm{s}$ to $1000 \mathrm{rad} / \mathrm{s}$.

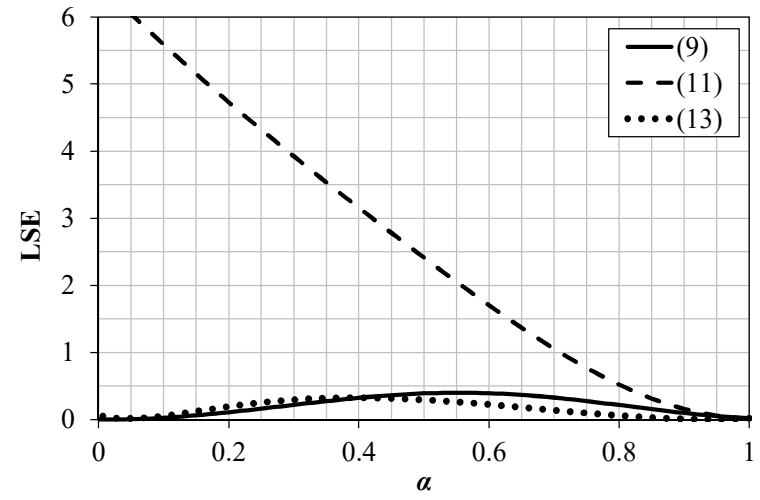

Fig. 3. LSE of the functions (9), (11), and (13) compared to the first-order function depending on $\alpha$. The frequency range is considered $1 \mathrm{rad} / \mathrm{s}$ to $1000 \mathrm{rad} / \mathrm{s}$.

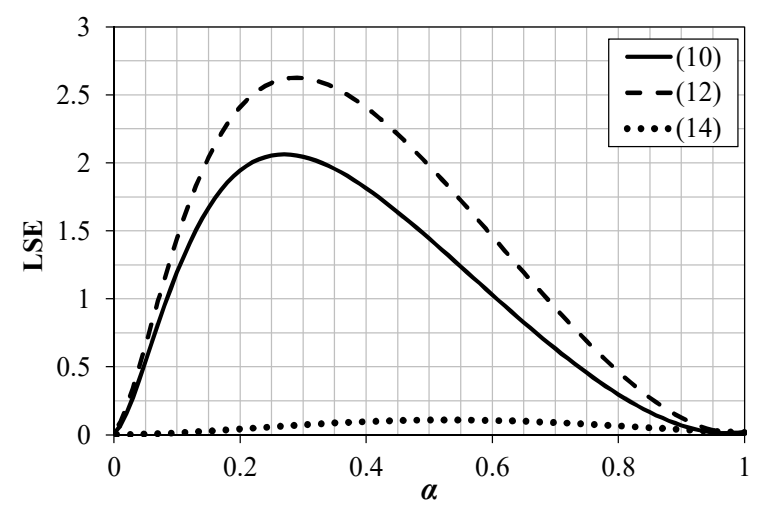

Fig. 4. LSE of the functions (10), (12), and (14) compared to the firstorder function depending on $\alpha$. The frequency range is considered $1 \mathrm{rad} / \mathrm{s}$ to $1000 \mathrm{rad} / \mathrm{s}$.

From Fig. 3, the function (13) provides the most accurate HP magnitude when compared to the first-order Butterworth HP response for $0.4<\alpha<1$. For $\alpha<0.4$ the function (9) shows slightly lower error than (13). It could be expected that when shifting the upper frequency limit for computing LSE above $1000 \mathrm{rad} / \mathrm{s}$ the transfer function (9) will provide the lowest LSE from the functions A. It is due to the already mentioned fact that the magnitude of (9) tends to $0 \mathrm{~dB}$ at high frequencies which is the same as the magnitude of the first-order HP function. In Fig. 4 it is clear that (14) is the most accurate for all $\alpha$ values considered, confirming the results earlier presented in Section II.

It is also useful to investigate the effect of the variations of the coefficients on the filter responses. Through a series of numerical simulations, it was noted that the coefficients $k_{\mathrm{A} 2}$ and $k_{\mathrm{B} 2}$ affect the magnitude of the HP functions (9)(14) at frequencies about $1 \mathrm{rad} / \mathrm{s}$. For example, when $k_{\mathrm{A} 2}$ or $k_{\mathrm{B} 2}$ are increased by $1 \mathrm{~dB}$, an increase of $12.2 \%$, the gain at $1 \mathrm{rad} / \mathrm{s}$ decreases by approximately $\alpha \mathrm{dB}$, while the gain at the stop- and pass-band frequencies remains almost 
unaffected. A $1 \mathrm{~dB}$ increase of $k_{\mathrm{A} 3}$ and $k_{\mathrm{B} 3}$ causes the magnitudes of (9) and (10) respectively to decrease by approx. $1 \mathrm{~dB}$ in the stop-band regardless of $\alpha$. In the case of (11) and (12) this coefficient change causes a decrease of $2 \mathrm{~dB}$ in the stop-band and $1 \mathrm{~dB}$ in the pass-band. Finally, the functions (13) and (14) react to this coefficient increase by decreasing their magnitude by approx. $1 \mathrm{~dB}$ in the passband.

\section{FRACTIONAL-ORDER BP TRANSFER FUNCTIONS}

The standard LP to BP transformation applied for integerorder filters results from replacing $s$ with $\left(s^{2}+1\right) /(s \cdot B)$, where $B$ is the desired bandwidth (in $\mathrm{rad} / \mathrm{s}$ ). However, this transformation is not applied here as it leads to a doubling of the filter order and an increased circuit complexity. Instead, two cases of FO BP transfer functions are obtained by multiplying the LP transfer functions (1) and (2) by the complex variable $s$ or $s^{\alpha}$. This limits the explored FO BP transfer functions in this work to only those with order $1+\alpha$.

\section{A. Case BP1}

By multiplying (1) and (2) by the complex variable $s$, the following BP transfer functions are obtained:

$$
\begin{gathered}
H_{1+\alpha}^{\mathrm{BP} 1-\mathrm{A}}(s)=\frac{s k_{\mathrm{A} 1}}{s^{1+\alpha}+s^{\alpha} k_{\mathrm{A} 2}+k_{\mathrm{A} 3}}, \\
H_{1+\alpha}^{\mathrm{BP} 1-\mathrm{B}}(s)=\frac{s k_{\mathrm{B} 1}}{s^{1+\alpha}+s k_{\mathrm{B} 2}+k_{\mathrm{B} 3}} .
\end{gathered}
$$

The transfer functions (1) and (16) appeared in [9] as the LP and BP responses at two outputs of a multi-loop feedback filter. While the functions (2) and (17) can be implemented by the Tow-Thomas filter as presented in [10].

The BP filters described by (16) and (17) provide stopband attenuations of $+20 \mathrm{~dB} / \mathrm{dec}$ and $-20 \alpha \mathrm{dB} / \mathrm{dec}$ for frequencies lower and higher, respectively, than the centre frequency. Thus, these transfer functions represent asymmetric BP magnitude responses with a stop-band slope at high frequencies that is dependent on the fractional component of the filter order.

\section{B. Case BP2}

Another method to obtain FO BP transfer functions multiplies (1) and (2) by the term $s^{\alpha}$ which yields:

$$
\begin{gathered}
H_{1+\alpha}^{\mathrm{BP} 2-\mathrm{A}}(s)=\frac{s^{\alpha} k_{\mathrm{A} 1}}{s^{1+\alpha}+s^{\alpha} k_{\mathrm{A} 2}+k_{\mathrm{A} 3}}, \\
H_{1+\alpha}^{\mathrm{BP} 2-\mathrm{B}}(s)=\frac{s^{\alpha} k_{\mathrm{B} 1}}{s^{1+\alpha}+s k_{\mathrm{B} 2}+k_{\mathrm{B} 3}} .
\end{gathered}
$$

Similar to the BP1 case, the functions (2) and (19) are realizable by the filter in [9] and the functions (1) and (18) by the Tow-Thomas filter in [10]. In contrast to the BP1 cases, the functions (18) and (19) feature stop-band attenuations of $+20 \alpha \mathrm{dB} / \mathrm{dec}$ and $-20 \mathrm{~dB} / \mathrm{dec}$ for frequencies lower and higher, respectively, than the centre frequency. In this case, providing a stop-band attenuation at low frequencies that is dependent on $\alpha$.

\section{EVALUATION OF THE BP TRANSFER FUNCTIONS}

To evaluate the BP transfer functions, the magnitude characteristics of each response compared to the secondorder Butterworth BP transfer function are presented in Fig. 5 and Fig. 6. Figure 5 presents the magnitude characteristics of (16) and (17) with $\alpha=0.5$ and the coefficients according to (3)-(8), with Fig. 6 depicting the magnitudes of (18) and (19), again with $\alpha=0.5$ and the coefficients according to (3)-(8). In this comparison, the second-order Butterworth BP transfer function was designed with a centre frequency of $1 \mathrm{rad} / \mathrm{s}$ and gain matched in the stop-band where the slopes are the same.

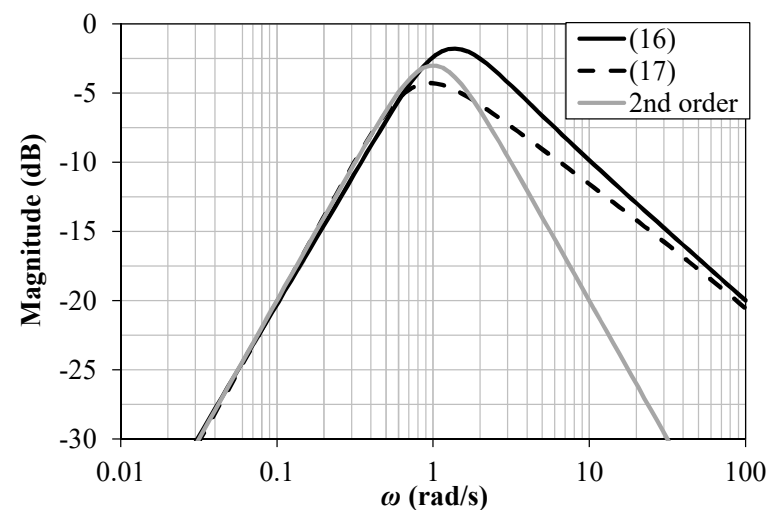

Fig. 5. Magnitude frequency characteristics of (16) and (17) with $\alpha=0.5$ compared to the second-order Butterworth BP response.

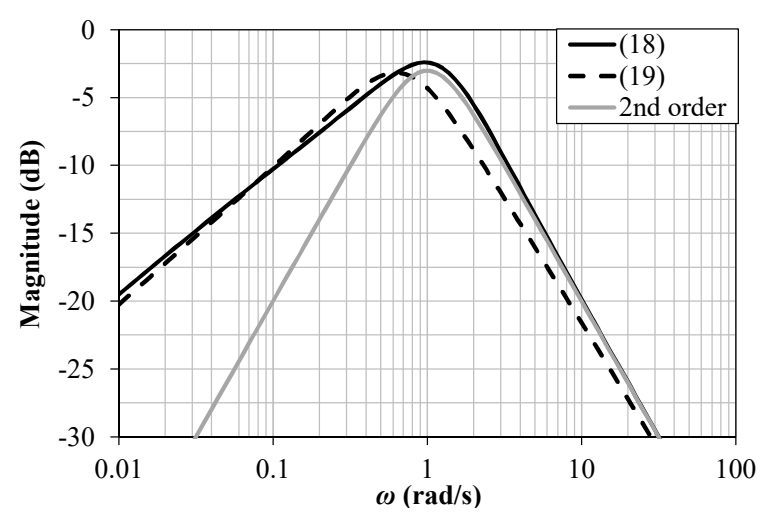

Fig. 6. Magnitude frequency characteristics of (18) and (19) with $\alpha=0.5$ compared to the second-order Butterworth BP response.

In Fig. 5 the gains of both FO filters and second-order Butterworth BP filter are almost identical below approximately $0.7 \mathrm{rad} / \mathrm{s}$. The magnitude of (16) shows both a higher centre frequency than (17) and higher gain from $0.7 \mathrm{rad} / \mathrm{s}$. As the frequency approaches $100 \mathrm{rad} / \mathrm{s}$ the magnitudes of (16) and (17) approach the expected $-10 \mathrm{~dB} / \mathrm{dec}$ attenuation.

Figure 6 reveals that the magnitude of (18) is well matched with the second-order Butterworth response above $1 \mathrm{rad} / \mathrm{s}$ and has almost the same centre frequency. On the other hand, the function (19) has a larger deviation from the second-order Butterworth BP characteristics in the upper stop-band, compared to (18), and has larger deviation of the centre frequency which occurs at $0.6 \mathrm{rad} / \mathrm{s}$.

Additionally, the LSE of the FO BP transfer functions compared to the second-order Butterworth BP response for $0<\alpha<1$ were calculated. The LSEs of the BP1 cases given by (16) and (17) were calculated comparing them to the second-order Butterworth BP from $0.01 \mathrm{rad} / \mathrm{s}$ to $1 \mathrm{rad} / \mathrm{s}$ 
using 100 datapoints; with the same process used for the BP2 cases given by (18) and (19) however in the range $1 \mathrm{rad} / \mathrm{s}$ to $100 \mathrm{rad} / \mathrm{s}$. Thus the error was determined only in the bands where the FO functions have a fixed slope that is independent of $\alpha$. These LSEs are presented in Fig. 7 and Fig. 8.

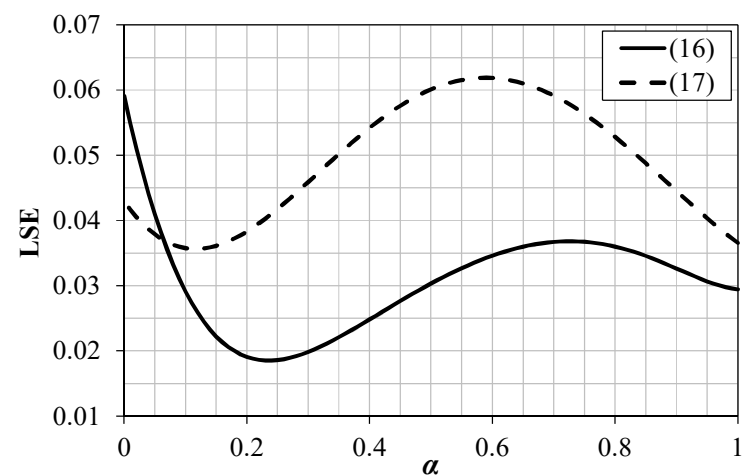

Fig. 7. LSE of the functions (16) and (17) compared to the second-order Butterworth function depending on $\alpha$. The frequency range is considered $0.01 \mathrm{rad} / \mathrm{s}$ to $1 \mathrm{rad} / \mathrm{s}$.

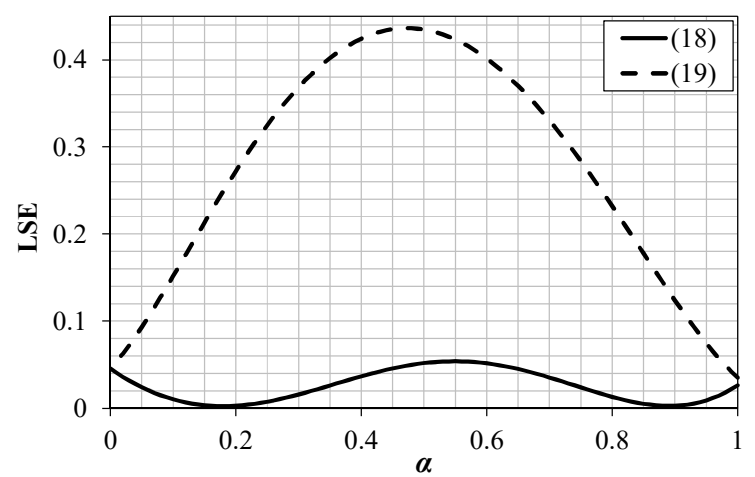

Fig. 8. LSE of the functions (18) and (19) compared to the second-order Butterworth function depending on $\alpha$. The frequency range is considered $1 \mathrm{rad} / \mathrm{s}$ to $100 \mathrm{rad} / \mathrm{s}$.

Based on Fig. 7 the function (16) provides lower error than (17) compared to the second-order Butterworth for $0.065<\alpha<1$. As seen in Fig. 8 the function (18) has comparable LSE to the functions (16) and (17) in Fig. 7. On the other hand, the function (19) displays the highest error reaching its maximum around $\alpha=0.5$. Based on these results, it is advisable to avoid using (19) with the coefficients from (6)-(8) to create a FO BP response which approximates the pass-band characteristics of the Butterworth response.

Also in the case of the BP responses, the influence of coefficient variations was investigated using numerical simulations. The increase of the coefficients $k_{\mathrm{A} 2}$ and $k_{\mathrm{B} 2}$ by $1 \mathrm{~dB}$ causes a decrease of the magnitude of (16)-(19) by approximately $\alpha \mathrm{dB}$ at frequencies about $1 \mathrm{rad} / \mathrm{s}$. The same change of $k_{\mathrm{A} 3}$ and $k_{\mathrm{B} 3}$ causes the gain to decrease by approx. $1 \mathrm{~dB}$ in the lower stop-band.

\section{CONCLUSIONS}

This work has presented multiple methods to generate $(1+\alpha)$ FO HP and BP transfer functions from their FO LP counterparts. The simulated magnitude characteristics of the generated FO HP and BP transfer functions have been compared to the ideal Butterworth responses to evaluate the most appropriate case to realize a FO response that still approximates the Butterworth response after the transformation. The presented LSE analyses suggest that in the frequency range between $1 \mathrm{rad} / \mathrm{s}$ and $100 \mathrm{rad} / \mathrm{s}$ the $\mathrm{FO}$ HP transfer functions (13) and (14) are the most appropriate to approximate the first-order Butterworth HP transfer functions using the previously determined LP coefficients. Additionally, from the FO BP transfer functions, (16)-(18) can be chosen without significant differences in their magnitude characteristics. This work has only explored how to realize the FO HP and BP responses using the transfer function coefficients previously determined from FO LP filters. Further works should investigate if more appropriate transfer function coefficients can be determined using the derived FO HP and BP transfer functions and optimization routines; which may provide a better alternative to designing these FO filters.

\section{REFERENCES}

[1] A. Radwan, A. Soliman, A. S. Elwakil, "First-order filters generalized to the fractional domain", J. Circuits Syst. Comput., vol. 17, no. 1, pp. 55-66, 2008. DOI: 10.1142/ S0218126608004162.

[2] A. Radwan, A. S. Elwakil, A. Soliman, "On the generalization of second-order filters to the fractional-order domain", J. Circuits Syst. Comput., vol. 18, no. 2, pp. 361-386, 2009. DOI: $10.1142 / \mathrm{S} 0218126609005125$.

[3] B. Maundy, A. Elwakil, T. Freeborn, "On the practical realization of higher-order filters with fractional stepping", Signal Processing, vol. 91, no. 3, pp. 484-491, 2011. DOI: 10.1016/j.sigpro.2010.06.018.

[4] T. Freeborn, "Comparison of $(1+\alpha)$ fractional-order transfer functions to approximate lowpass Butterworth magnitude responses", Circuits, Systems, and Signal Processing, vol. 35, no. 6, pp. 1983-2002, 2016. DOI: 10.1007/s00034-015-0226-y.

[5] T. J. Freeborn, B. Maundy, A. S. Elwakil, "Approximated fractional order Chebyshev lowpass filters", Mathematical Problems in Engineering, 2014. DOI: 10.1155/ 2014/832468.

[6] T. J. Freeborn, A. S. Elwakil, B. Maundy, "Approximated fractionalorder inverse Chebyshev lowpass filters", Circuits, Systems, and Signal Processing, vol. 35, no. 6, pp. 1973-1982, 2016. DOI: 10.1007/s00034-015-0222-2.

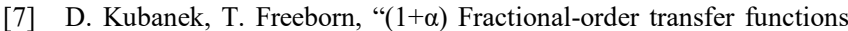
to approximate low-pass magnitude responses with arbitrary quality factor", International Journal of Electronics and Communications $(A E U)$, vol. 83, pp. 570-578, 2018. DOI: 10.1016/j.aeue.2017.04.031.

[8] P. Bertsias, F. Khateb, D. Kubanek, F. A. Khanday, C. Psychalinos, "Capacitorless digitally programmable fractional-order filters", International Journal of Electronics and Communications (AEU), vol. 78, pp. 228-237, 2017. DOI: 10.1016/j.aeue.2017.04.030.

[9] D. Kubanek, J. Koton, J. Jerabek, P. Ushakov, A. Shadrin, "Design and properties of fractional-order multifunction filter with DVCCs", in 39th Int. Conf. Telecommunications and Signal Processing (TSP 2016), Vienna, 2016, pp. 620-624. DOI: 10.1109/TSP.2016.7760956.

[10] T. J. Freeborn, B. Maundy, A. S. Elwakil, "Fractional-step TowThomas biquad filters", Nonlinear Theory Appl., vol. 3, no. 3, pp. 357-374, 2012. DOI: $10.1587 /$ nolta.3.357.

[11] A. Elwakil, "Fractional-order circuits and systems: An emerging interdisciplinary research area", IEEE Circuits and Systems Magazine, vol. 10, no. 4, pp. 40-50, 2010. DOI: 10.1109/MCAS.2010.938637.

[12] M. D. Ortigueira, "An introduction to the fractional continuous time linear systems: The 21st century systems", IEEE Circuits Syst. Mag., vol. 8, pp. 19-26, 2008. DOI: 10.1109/MCAS.2008.928419. 